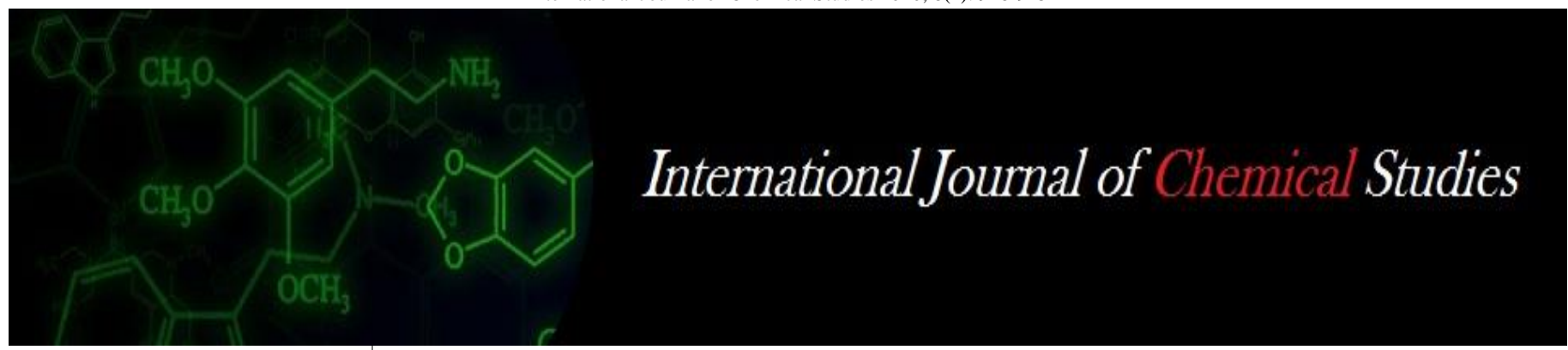

P-ISSN: 2349-8528

E-ISSN: 2321-4902

IJCS 2020; 8(1): 918-925

(C) 2020 IJCS

Received: 16-11-2019

Accepted: 20-12-2019

Tusar Ranjan Sahoo

Krishi Vigyan Kendra, ICAR-

National Rice Research

Institute, Cuttack, Odisha, India

Dinesh Kumar Singh

Gobind Ballav Pant University

of Agriculture and Technology,

Pantnagar, Uttarakhand, India

Corresponding Author:

Tusar Ranjan Sahoo

Krishi Vigyan Kendra, ICAR-

National Rice Research

Institute, Cuttack, Odisha, India

\section{Exploitation of heterosis in cucumber for earliness, yield and yield contributing traits under protected structure}

\section{Tusar Ranjan Sahoo and Dinesh Kumar Singh}

DOI: https://doi.org/10.22271/chemi.2020.v8.i11.8367

\begin{abstract}
The research was conducted at Vegetable Research Centre, Gobind Ballav Pant University of Agriculture and Technology, Pantnagar, Uttarakhand during 2015-16. Fourteen parental lines including eight parthenocarpic lines, three gynoecious lines and three testers of monoecious lines were used to develop $33 \mathrm{~F}_{1}$ hybrids of cucumber by Line $\times$ Tester mating design to determine the magnitude of heterosis for earliness, yield and yield related traits during March-June and August -December in protected structures. The mean sum of squares were highly significant for all the characters indicated a wide genetic variation for the characters studied and the Parthenocarpic parental lines (PCUCP-2, PCUCP-3 and PCUCP-8) and monoecious parent (Pant Khira-1, PCUC-8, and PCUC-25) were observed to be the three top performing parents for yield per plant. Appreciable heterosis was observed over better parent and standard parent for most of the characters studied. The best $\mathrm{F}_{1}$ hybrid PCUCP- $4 \times$ PCUC- 8 , promising in both E1 and E2 showing $835 \%$ and $453 \%$ heterosis, respectively for yield per plant followed by PCUCP-3 x PCUC-8 $(769 \%, 559 \%)$ for average fruit weight and PCUCP-1 $\times$ PCUC-8 $(-98.77 \%,-95.53 \%)$ for node number to first female flower over standard check Pant Khira-1. So, PCUCP-4 $\times$ PCUC- 8 can be tested as most stable genotype across the spring -summer and kharif-winter environments under polyhouse condition in northern plains and three hybrids viz., PCUCP- $4 \times$ PCUC- 8, PCUCP- $3 \times$ PCUC- 8 and PCUCP- $1 \times$ PCUC-8 may be exploited for commercial cultivation.
\end{abstract}

Keywords: Cucumber, boron, yield, quality, konkan

\section{Introduction}

Cucumber (Cucumis sativus L.) belongs to family Cucurbitaceae is an important summer vegetable, grown for its immature fruits, used as salad, making pickles, rayata preparations and even brined on commercial scale in almost every part of the world. Cucumbers with attractive fruit color, high total soluble solids content, less bitterness and high nutritive value are preferred by the consumer (Kumar et al., 2016) ${ }^{[17]}$. It is primarily a monoecious, crosspollinated crop and produces large number of seeds per pollination, which provides ample scope for the utilization of heterosis breeding and has a great scope of improvement over its base population.

For developing superior varieties, it is necessary to improve the earliness and yield in cucumber. This can be achieved through effective utilization of germplasm resources and integration of genomic tools to impart efficiency and pace of breeding processes. Using best combiners, heterosis breeding is one of the best methods to improve upon the existing varieties. India being considered the home of cucumber possesses a vast range of genetic diversity and variability for both growth and fruit characters, but this advantage has not been fully assessed and utilized. Heterosis breeding can be exploited as most efficient tools to exploit the genetic diversity in many cucurbitaceous crops including pumpkin (Mohanty and Mishra, 1999) [21]. Heterosis breeding provides an opportunity for achieving unique improvement in yield and other desirable attributes in one generation that would be more time consuming and difficult with other conventional breeding methods (Sherpa et al., 2014) ${ }^{[26]}$. Among many cucurbits grown across the world, cucumber is distinct with a unique sex mechanism and this feature can easily be manipulated for the production of F1 hybrid seeds (Arinia et al., 2013) ${ }^{[1]}$. Several breeders have confirmed that hybrid vigour was manifested in cucumber in respect of earliness (Hutchins, 1939) ${ }^{[11]}$ and increased yield due to large number of fruits per plant (Singh et al., 1970; Pandey et al., 2005 and Airina et al., 2013) ${ }^{[1,29,24]}$. 
Heterosis is rather a function of specific cross combination, so analysis of combining ability helps to determine the feasibility of its utilization and identification of best combiners. It also helps in the identification of superior hybrid combinations, which may be utilized for commercial exploitation of heterosis (Reddy et al., 2014) ${ }^{[25]}$. Therefore, there is a paramount need to develop suitable hybrids, which may be utilized on commercial scale especially in the north Indian plains. Temperature plays a vital role in production and productivity of cucumber in open condition and protected structures as well. It is also important to develop suitable stable lines under protected structures to be grown under polyhouse round the year.

Keeping in view the above facts, the present investigation was therefore, initiated with a view to obtain suitable hybrids which can be exploited on commercial scale in the north Indian conditions so the information for assessment of heterosis for earliness, yield and yield attributing traits has been evaluated.

\section{Materials and Methods}

The present experiment was carried out at Vegetable Research Centre, Gobind Ballav Pant University of Agriculture and Technology, Pantnagar during 2015 and 2016.The fourteen genetically diverse genotypes of cucumber viz., eight were parthenocarpic, three were from gynoecious types were crossed with three monoecious genotypes in Line $\times$ Tester mating design and $33 \mathrm{~F}_{1}$ hybrids were obtained. These $33 \mathrm{~F}_{1}$ 's along with the fourteen parents were evaluated in an experiment in randomized complete block design (RCBD) with three replications in two environments under polyhouse. One set was during March-June sowing as E1 and other set was during August-December sowing as E2. The crop was grown in rows at $0.60 \mathrm{~m}$ apart with spacing of $0.60 \mathrm{~m}$ between the plants. All the recommended agronomic practices including weeding, hoeing, manures and fertigations were followed to raise a healthy and successful crop (Anonymous, 2016) ${ }^{[2]}$. In each replication per plot, out of nine plants, five plants were randomly selected for observations on plant growth, and yield attributing characters on individual plant basis. The observations were recorded for important characters namely, days taken to first female flower anthesis, node number to first female flower, Days to first fruit harvest, vine length $(\mathrm{m})$, fruit length $(\mathrm{cm})$, fruit diameter $(\mathrm{cm})$, average fruit weight $(\mathrm{g})$, number of fruits per plant, fruit yield per plant $(\mathrm{kg})$ and fruit yield per hectare $(\mathrm{q})$. Therefore, heterosis was calculated in favourable direction as percentage increase of $\mathrm{F}_{1}$ performance over better parent (BP) and standard check (SC). Increase or decrease was measured as the proportion of deviation of $\mathrm{F}_{1}$ from better parent (heterobeltiosis) and standard parent Pant Khira-1 (check) and expressed in percentage.

1. Heterosis over better parent $(\mathrm{BP})=\left[\left(\mathrm{F}_{1}-\mathrm{BP}\right) / \mathrm{BP}\right] \times 100$

2. Heterosis over standard check $(\mathrm{SC})=\left[\left(\mathrm{F}_{1}-\mathrm{SC}\right) / \mathrm{SC}\right] \times$ 100

\section{The standard error was calculated as under}

SE $(d)= \pm(2 \mathrm{Me} / \mathrm{r})$ In order to test the significance of heterosis over BP and increase/decrease over check, t-test as follows was conducted:

1. ' $t$ ' calculated values for heterosis over $B P=\left(F_{1}\right.$ $\mathrm{BP}) / \mathrm{SE}(\mathrm{d})$

2. ' $\mathrm{t}$ ' calculated values for heterosis over $\mathrm{SC}=\left(\mathrm{F}_{1}\right.$ $\mathrm{SC}) / \mathrm{SE}(\mathrm{d})$
If the calculated t-value was greater than tabulated value at error degree of freedom and at least significant difference then only the results were declared significant (Hayes, 1955 and Turner, 1953) ${ }^{[9,31]}$.

\section{Results and Discussion}

There were highly significant differences among the genotypes in respect of different characters studied including total yield per plant and per hectare. The range of mean performance of parents, crosses and check variety for earliness and yield contributing traits is presented in Table 1 and Table 2. Earliness, indicated by negative estimates of heterosis which helps the grower to fetch early market price, is a well recognised and one of the most important desirable parameter in any breeding programme particularly development of hybrids. This trait is associated with characters such as days taken to first female flower anthesis, node number to first female flower, days to first fruit harvest. In order of superiority, the best four F1 hybrids, which gave best performance over better parent in relation to earliness were, PCUCP- $2 \times$ PCUC- 8 (-19.45\%), PCUCP- $8 \times$ PCUC-25 $(-13.20 \%)$, PCUCP-2 $\times$ PCUC-25 (-11.64\%) and PCUCP-1 $\times$ PCUC-8 $(-10.55 \%)$ for days taken to first female flower anthesis in environment 1; PCUCP-4 × PCUC-25 (-22.11\%), PCUCP-1× Pant Khira-1 (-21.67\%), PCUCP-1 $\times$ PCUC-25 ($19.55 \%)$ and PCUCP-4 $\times$ PCUC-8 $(-17.92 \%)$ for days taken to first female flower anthesis in environment 2 ; PGYC-3 $\times$ PCUC-25 (-53.66\%), PCUCP-1 × PCUC-8 (-17.72\%) for node number to first female flower in environment 1 ; PGYC$1 \times$ PCUC-8 (-60.78\%), PGYC-1 × PCUC-25 (-51.63\%), PCUCP-2 $\times$ Pant Khira-1 (-51.56\%) and PCUCP-1 $\times$ PCUC- 8 $(-41.77 \%)$ for node number to first female flower in environment 2; PCUCP-5 $\times$ Pant Khira-1 (-21.95\%), PGYC-1 $\times$ Pant Khira-1 (-21.61\%), PGYC-1 $\times$ PCUC-8 (-21.11\%), PGYC-1 $\times$ PCUC-25 (-14.07\%) for days to first harvest in environment 1; PGYC-1 $\times$ PCUC-8 (-17.62\%), PGYC-3 $\times$ PCUC-25 (-14.68\%), PGYC-1 $\times$ Pant Khira-1 (-11.43\%) and PCUCP-5 $\times$ PCUC-8 $(-7.49 \%)$ for days to first harvest in environment 2; PCUCP-1 $\times$ PCUC-8 (142.6\%), PCUCP-7 $\times$ PCUC-25 (113.7\%), PCUCP-1 $\times$ Pant Khira-1 (102.6\%) and PCUCP-8 $\times$ Pant Khira-1 $(90.0 \%)$ for vine length in environment 1; PCUCP-1 × PCUC-8 (213.79\%), PCUCP1×Pant Khira-1 (168.97\%), PCUCP-1 × PCUC-25 (97.59\%) and PCUCP-7 $\times$ PCUC-25 $(88.33 \%)$ for vine length in environment 2 . Based on two environments under polyhouse for trait like node number to first female flower anthesis, the cross combination PCUCP- $1 \times$ PCUC- 8 is more stable for giving better parent heterosis. Similarly, PCUCP- $1 \times$ PCUC- 8 and PCUCP- $8 \times$ PCUC-25 were more stable for giving standard heterosis for the above trait. But no cross combination exhibited its stability in both environments for days to first female flower anthesis. (Table 3 and Table 4). Wide variations with respect to earliness were also reported by Munshi et al. (2007), Kumar et al. (2008), Hanchinamani et al. (2009), Yadav et al. (2009), Kumar et al. (2010), Singh et al. (2010), Dogra and Kanwar (2011), Kumar et al. (2013), Airina et al. (2013), Jat et al. (2015) and Kumar et al. (2017) in cucumber $[1,4,7,12,13,14,15,16,17,23,32]$.

Highest yield is the foremost and desirable character for any breeding programme. It is a complex trait resulting from the interaction of its component character of a crop. Moll and Stuber (1974) ${ }^{[22]}$ pointed out that heterosis estimates should indicate whether heterozygote's or homozygote's represent the more ideal genotype. In case of cucumber breeding, 
number of fruits per plant, fruit weight and fruit size are the direct yield components.

In order of merit the best four $F_{1}$ hybrids, which gave highest performance over better parent in relation to yield and its contributing characters were PCUCP-8 $\times$ Pant Khira-1 $(54.56 \%)$, PCUCP-8 $\times$ PCUC-8 $(45.67 \%)$, PCUCP-4 $\times$ PCUC-8 (41.98\%) and PGYC-2 $\times$ PCUC-28 (39.93\%) for fruit length in environment 1; PGYC-2 $\times$ Pant Khira-1 $(41.18 \%)$, PGYC-2 $\times$ PCUC-25 $(39.48 \%)$, PCUCP-4 $\times$ PCUC-8 (35.48\%) and PCUCP-1 $\times$ PCUC-25 (39.40\%) for fruit length in environment 2; PGYC-2 $\times$ Pant Khira-1 (27.82\%), PCUCP-3 × PCUC-8 (18.60\%), PGYC-3 $\times$ Pant Khira-1 (17.23\%) and PGYC-3 $\times$ PCUC-8 (15.32\%) for fruit diameter in environment 1 ; PGYC-1 $\times$ Pant Khira-1 (34.09\%), PGYC-1 × PCUC-25 (30.32\%), PCUCP-2 $\times$ Pant Khira-1 (21.08\%) and PGYC-3 $\times$ Pant Khira-1 (16.59\%) for fruit diameter in environment 2 ; PCUCP-5 $\times$ PCUC-8 (97.22\%), PCUCP-5 × PCUC-25 $(68.06 \%)$, PCUCP-4 $\times$ PCUC-8 (65.69\%) and PCUCP-5 × Pant Khira-1 $(33.33 \%)$ for number of fruits per plant in environment 1 ; PCUCP-4 $\times$ PCUC-8 (79.25\%), PCUCP-5 × PCUC-8 (62.39\%), PCUCP-5 $\times$ Pant Khira-1 (51.38\%) and PCUCP-4 × PCUC-25 (42.14\%) for number of fruits per plant in environment 2; PGYC-1 $\times$ Pant Khira-1 (100.32\%), PGYC-1 × PCUC-25 (84.01\%), PCUCP-6 $\times$ PCUC-25 $(82.05 \%)$ and PCUCP-6 $\times$ Pant Khira$1(81.99 \%)$ for average fruit weight in environment 1 ; PCUCP-6 $\times$ Pant Khira-1 (79.25\%), PCUCP-6 × PCUC-25 (70.90\%), PCUCP-8 $\times$ Pant Khira-1 $(66.61 \%)$ and PCUCP-1 $\times$ PCUC-25 $(57.71 \%)$ for average fruit weight in environment 2; PCUCP-6 × PCUC-25(170.61\%), PCUCP-5 × PCUC8(152.88\%), PGYC-1 $\times$ Pant Khira-1(139.32\%) and PCUCP$6 \times$ PCUC-8 $(89.47 \%)$ for yield per plant in environment 1 ; PCUCP-5 $\times$ PCUC-8 (156.24\%), PCUCP-1 $\times$ Pant Khira-1 (119.45\%), PCUCP-1 x PCUC-25 (108.84\%), PCUCP-4 $\times$ PCUC-8 $(89.97 \%$ ) for yield per plant in environment 2 (Table 3 and Table 4). These findings were in line with Munshi et al. (2007), Kumar et al. (2008), Hanchinamani et al. (2008), Yadav et al. (2009), Kumar et al. (2010), Singh et al. (2010), Hossain et al. (2010), Dogra and Kanwar (2011), Kumar et al. (2011), Golabadi et al. (2012), Singh et al. (2012), Airina et al. (2013), Jat et al. (2015), Kumar et al. (2017) and Thakur et al. (2017), who have also been reported wide variations with respect to yield and yield contributing traits in cucumber ${ }^{[23,4,5,1,7,10,12,13,14,15,16,17,23,28,30,32 \mathrm{~s}]}$.

The result indicated that maximum yield per plant in the hybrids mentioned above was attributed by maximum number of fruits per plant. First generation crosses in cucumber frequently exhibit high parent heterosis due to increase fruit size and fruit number per plant was reported by Hayes and Jones (1916) ${ }^{[8]}$. Hence, breeder should concentrate mainly on fruit number rather than fruit size in their efforts to increase yield. The present experiment showed a fairly high degree of heterosis for fruit yield per plant and per hectare in most of hybrids. Singh et al. (2012) ${ }^{[28]}$ observed positive heterosis desirable for length of fruit, weight per fruit, number of fruits per plant and fruit yield per plant was common in most of the crosses. Vijayakumari et al. (1991) [33] reported that gynoecious and monoecious hybrids were promising for earliness. Kushwaha et al. (2011) ${ }^{[19]}$ observed that hybrids manifested significant heterobeltiosis for node bearing first female flower, fruit length, fruit diameter, fruit weight and for number of fruits per vine and fruit yield per vine. Bairagi et al. (2002) ${ }^{[3]}$ reported promising heterosis for fruit yield and yield contributing traits in cucumber. Musmade (1986) [20] also reported similar results in cucumber hybrids. Appreciable heterosis in desirable direction was found over better parent and mid parent for all the characters studied (Pandey et al., 2005) [24]. In accordance to the present findings, Grafius (1959) ${ }^{[6]}$ was of the opinion that hybrid vigour of even small magnitude of individual yield components may have additive or synergistic effect on the end product, as had mentioned that heterosis for yield is the result of interaction of simultaneous increase in the expression of heterosis for yield components. Based on the performance of $33 \mathrm{~F} 1$ hybrids three best performing hybrids under environment 1 were PCUCP-3 $\times$ PCUC-8, PCUCP-1 $\times$ PCUC-25 and PCUCP- $4 \times$ PCUC-8 showing 559, 486 and $453 \%$ heterosis, respectively over standard check Pant Khira-1 for yield per plant and per hectare (Table 5). Similarly three best performing hybrids under environment 2 were PCUCP-3 $\times$ PCUC-25, PCUCP-4 $\times$ PCUC- 8 and PCUCP-1 $\times$ Pant Khira-1 showing 937, 835 and $769 \%$ heterosis respectively over standard check Pant Khira-1 for yield per plant and per hectare (Table 6) can be tested under multi-locational trials so that these hybrids can be further utilized for commercial scale under protected condition. In both the environments, PCUCP- $4 \times$ PCUC- 8 showed its stable performance for its superiority over standard parent for yield per plant and hectare.

It can be concluded that the $F_{1}$ hybrids those found to be superior in performance in both the environments (E1 and E2) over standard parents for various characters were PCUCP- $4 \times$ PCUC-8 for number of fruits per plant, fruit yield per plant and fruit yield per hectare, PCUCP-3 3 PCUC-25 for internodal length and fruit diameter, PCUCP $-1 \times$ PCUC- 8 for node number to first female flower, PCUCP- $3 \times$ PCUC- 8 for average fruit weight, PCUCP- $4 \times$ PCUC-25 for days to first fruit harvest. The best $\mathrm{F}_{1}$ hybrid PCUCP-4 $\times$ PCUC-8, promising in both $\mathrm{E} 1$ and $\mathrm{E} 2$ showing $835 \%$ and $453 \%$ heterosis, respectively for yield per plant followed by PCUCP-3 x PCUC-8 $(769 \%, 559 \%)$ for average fruit weight and PCUCP-1× PCUC-8 (-98.77\%,-95.53\%) for node number to first female flower over standard check Pant Khira-1. So, PCUCP- $4 \times$ PCUC -8 can be tested as most stable genotype across the spring -summer and kharif-winter environments under polyhouse condition in northern plains and these three hybrids viz., PCUCP-4 $\times$ PCUC- 8 , PCUCP- $3 \times$ PCUC- 8 and PCUCP-1× PCUC-8 may be exploited for commercial cultivation.

Table 1: Mean performance of parents and $F_{1}$ hybrids for important quantitative traits in $E 1$

\begin{tabular}{|c|c|c|c|c|c|c|c|c|c|c|}
\hline Genotypes & DFFFA & NNFFF & DFFH & VL $(\mathbf{m})$ & FL $(\mathbf{c m})$ & FD $(\mathbf{c m})$ & NF/ P & AFW $(\mathbf{g})$ & FY/P (kg) & FY/Ha (q) \\
\hline PCUCP-1 & 44.87 & 5.27 & 62.67 & 1.95 & 15.21 & 3.40 & 10.00 & 144.00 & 1.84 & 485.77 \\
\hline PCUCP-2 & 48.67 & 4.20 & 60.67 & 4.10 & 15.52 & 3.67 & 12.20 & 148.83 & 2.38 & 628.53 \\
\hline PCUCP-3 & 46.93 & 6.20 & 58.67 & 2.33 & 19.64 & 3.80 & 9.20 & 154.74 & 1.88 & 497.99 \\
\hline PCUCP-4 & 40.00 & 3.93 & 58.00 & 2.63 & 12.34 & 4.74 & 6.80 & 175.52 & 1.63 & 429.71 \\
\hline PCUCP-5 & 36.00 & 4.00 & 68.33 & 3.20 & 12.36 & 4.52 & 4.80 & 150.28 & 0.93 & 243.41 \\
\hline PCUCP-6 & 43.07 & 4.27 & 61.00 & 2.03 & 15.24 & 4.23 & 5.80 & 110.26 & 0.76 & 200.30 \\
\hline PCUCP-7 & 40.60 & 2.27 & 52.00 & 2.17 & 15.28 & 4.14 & 7.33 & 170.95 & 1.70 & 447.65 \\
\hline
\end{tabular}




\begin{tabular}{|c|c|c|c|c|c|c|c|c|c|c|}
\hline PCUCP-8 & 44.93 & 3.27 & 56.33 & 2.00 & 12.00 & 3.80 & 12.67 & 130.00 & 2.60 & 556.37 \\
\hline PGYC-1 & 36.80 & 3.40 & 66.33 & 2.67 & 12.20 & 3.95 & 6.00 & 85.00 & 0.69 & 179.35 \\
\hline PGYC-2 & 40.93 & 5.33 & 58.33 & 4.92 & 15.23 & 4.03 & 6.20 & 168.61 & 1.36 & 359.98 \\
\hline PGYC-3 & 40.13 & 8.20 & 67.67 & 4.11 & 13.65 & 4.53 & 6.80 & 162.42 & 1.53 & 403.10 \\
\hline PCUC-8 & 68.55 & 18.60 & 78.00 & 2.95 & 16.24 & 4.45 & 2.00 & 198.00 & 0.42 & 110.43 \\
\hline PCUC-25 & 69.87 & 27.00 & 81.33 & 2.75 & 18.00 & 4.22 & 2.00 & 190.00 & 0.38 & 104.21 \\
\hline Pant Khira-1 & 71.87 & 17.60 & 81.67 & 3.92 & 18.70 & 4.10 & 2.00 & 200.00 & 0.46 & 121.39 \\
\hline PCUCP-1×PCUC-8 & 40.13 & 4.33 & 56.67 & 4.73 & 15.40 & 3.63 & 7.00 & 156.83 & 1.47 & 388.78 \\
\hline PCUCP-1×PCUC-25 & 51.33 & 11.27 & 65.00 & 2.86 & 16.50 & 3.81 & 9.13 & 212.15 & 2.69 & 709.69 \\
\hline PCUCP-1×Pant Khira-1 & 46.47 & 7.27 & 65.67 & 3.95 & 16.05 & 3.34 & 9.87 & 185.96 & 2.49 & 656.01 \\
\hline PCUCP-2×PCUC-8 & 39.20 & 4.00 & 56.00 & 4.97 & 16.22 & 3.57 & 8.60 & 162.70 & 1.77 & 466.39 \\
\hline PCUCP-2×PCUC-25 & 43.00 & 5.20 & 57.67 & 3.85 & 14.55 & 3.60 & 8.73 & 149.22 & 1.84 & 486.14 \\
\hline PCUCP-2×Pant Khira-1 & 51.40 & 11.13 & 67.67 & 2.97 & 15.61 & 4.69 & 7.93 & 174.37 & 1.75 & 461.79 \\
\hline PCUCP-3×PCUC-8 & 49.00 & 7.80 & 59.67 & 3.35 & 20.11 & 4.51 & 9.13 & 240.17 & 3.03 & 803.88 \\
\hline PCUCP-3×PCUC-25 & 43.53 & 6.13 & 57.67 & 2.85 & 16.54 & 3.24 & 8.13 & 166.96 & 1.85 & 490.70 \\
\hline PCUCP-3×Pant Khira-1 & 48.00 & 10.47 & 59.00 & 3.92 & 14.56 & 3.20 & 6.27 & 160.84 & 1.45 & 385.37 \\
\hline PCUCP-4×PCUC-8 & 46.00 & 4.53 & 60.00 & 4.17 & 17.52 & 3.82 & 11.27 & 176.25 & 2.54 & 670.79 \\
\hline PCUCP-4×PCUC-25 & 41.07 & 4.87 & 52.00 & 3.67 & 14.20 & 4.15 & 8.07 & 164.95 & 1.79 & 474.79 \\
\hline PCUCP-4×Pant Khira-1 & 37.00 & 5.00 & 56.33 & 3.63 & 16.29 & 4.80 & 6.40 & 212.79 & 1.97 & 520.88 \\
\hline PCUCP-5×PCUC-8 & 42.87 & 4.80 & 61.67 & 4.41 & 16.27 & 3.74 & 9.47 & 162.27 & 2.34 & 618.68 \\
\hline PCUCP-5×PCUC-25 & 42.93 & 6.00 & 65.67 & 2.50 & 13.50 & 3.70 & 8.07 & 150.83 & 1.47 & 387.57 \\
\hline PCUCP-5×Pant Khira-1 & 43.07 & 5.93 & 53.33 & 2.05 & 13.64 & 3.74 & 6.40 & 190.13 & 1.70 & 449.57 \\
\hline PCUCP-6×PCUC-8 & 46.33 & 6.07 & 58.00 & 3.25 & 13.54 & 4.40 & 6.80 & 142.47 & 1.44 & 379.66 \\
\hline PCUCP-6×PCUC-25 & 47.13 & 8.07 & 58.00 & 2.77 & 14.52 & 4.58 & 7.53 & 200.73 & 2.06 & 550.80 \\
\hline PCUCP-6×Pant Khira-1 & 45.47 & 8.27 & 57.67 & 3.78 & 18.74 & 4.50 & 7.53 & 200.67 & 1.85 & 520.29 \\
\hline PCUCP-7×PCUC-8 & 42.87 & 8.93 & 52.33 & 3.86 & 16.52 & 3.53 & 9.60 & 169.83 & 2.34 & 607.09 \\
\hline PCUCP-7×PCUC-25 & 51.33 & 10.87 & 62.00 & 4.63 & 18.95 & 4.29 & 6.20 & 214.21 & 1.97 & 518.74 \\
\hline PCUCP-7×Pant Khira-1 & 54.00 & 13.47 & 59.33 & 4.00 & 16.50 & 3.43 & 6.20 & 102.28 & 0.85 & 233.79 \\
\hline PCUCP-8×PCUC-8 & 46.00 & 6.87 & 52.33 & 3.70 & 17.48 & 3.65 & 6.80 & 179.96 & 1.82 & 479.96 \\
\hline PCUCP-8×PCUC-25 & 39.00 & 4.00 & 57.33 & 4.27 & 15.25 & 4.01 & 8.00 & 143.92 & 1.62 & 428.15 \\
\hline PCUCP-8×Pant Khira-1 & 45.47 & 6.00 & 60.67 & 3.80 & 18.55 & 3.67 & 4.13 & 170.32 & 0.97 & 258.45 \\
\hline PGYC-1×PCUC-8 & 40.00 & 4.00 & 52.33 & 4.50 & 13.55 & 3.83 & 5.33 & 152.41 & 1.23 & 324.42 \\
\hline PGYC-1×PCUC-25 & 38.47 & 5.00 & 57.00 & 4.27 & 13.55 & 4.00 & 6.73 & 156.41 & 1.22 & 324.31 \\
\hline PGYC-1×Pant Khira-1 & 40.33 & 7.00 & 52.00 & 3.82 & 14.00 & 4.10 & 6.80 & 170.27 & 1.64 & 433.04 \\
\hline PGYC-2×PCUC-8 & 45.20 & 10.20 & 75.00 & 4.16 & 19.50 & 4.00 & 5.40 & 168.92 & 1.27 & 336.86 \\
\hline PGYC-2×PCUC-25 & 45.47 & 7.00 & 64.33 & 4.17 & 21.22 & 4.25 & 6.20 & 172.68 & 1.58 & 419.12 \\
\hline PGYC-2×Pant Khira-1 & 68.47 & 18.33 & 77.33 & 3.83 & 21.32 & 4.36 & 8.00 & 170.00 & 1.78 & 470.09 \\
\hline PGYC-3×PCUC-8 & 45.00 & 9.13 & 65.33 & 6.20 & 13.44 & 5.22 & 5.60 & 150.34 & 1.40 & 367.35 \\
\hline PGYC-3×PCUC-25 & 54.20 & 3.80 & 71.67 & 4.60 & 12.24 & 4.23 & 6.00 & 196.93 & 1.75 & 460.34 \\
\hline PGYC-3×Pant Khira-1 & 61.00 & 18.80 & 70.33 & 4.65 & 14.00 & 5.31 & 6.20 & 210.32 & 1.78 & 470.35 \\
\hline Mean & 46.68 & 7.83 & 61.87 & 3.61 & 15.77 & 4.05 & 7.18 & 168.67 & 1.65 & 432.38 \\
\hline C.V. & 1.38 & 6.42 & 1.37 & 8.24 & 0.92 & 2.84 & 11.15 & 5.51 & 9.97 & 4.50 \\
\hline S.E. & 0.37 & 0.29 & 0.49 & 0.17 & 0.08 & 0.07 & 0.46 & 5.36 & 0.09 & 11.25 \\
\hline C.D. $5 \%$ & 1.05 & 0.82 & 1.37 & 0.48 & 0.24 & 0.19 & 1.30 & 15.06 & 0.27 & 31.59 \\
\hline C.D. $1 \%$ & 1.39 & 1.08 & 1.82 & 0.64 & 0.31 & 0.25 & 1.72 & 19.95 & 0.35 & 41.83 \\
\hline
\end{tabular}

Table 2: Mean performance of parents, F1 hybrids for important quantitative traits in E2

\begin{tabular}{|c|c|c|c|c|c|c|c|c|c|c|}
\hline Genotypes & DFFFA & NNFFF & DFFH & VL $(\mathbf{m})$ & FL (cm) & FD $(\mathbf{c m})$ & NF/P & AFW $(\mathbf{g})$ & FY /P (kg) & FY/Ha (q) \\
\hline PCUCP-1 & 47.07 & 5.27 & 57.67 & 1.93 & 12.50 & 3.50 & 12.00 & 140.00 & 2.07 & 548.56 \\
\hline PCUCP-2 & 33.33 & 4.27 & 58.00 & 4.21 & 18.05 & 3.70 & 14.80 & 172.29 & 3.17 & 832.72 \\
\hline PCUCP-3 & 35.93 & 6.00 & 60.00 & 3.20 & 22.36 & 3.84 & 12.40 & 183.62 & 3.02 & 790.93 \\
\hline PCUCP-4 & 46.13 & 5.00 & 57.67 & 2.72 & 13.02 & 4.70 & 10.60 & 175.04 & 2.46 & 650.09 \\
\hline PCUCP-5 & 40.33 & 2.93 & 62.33 & 4.00 & 12.43 & 4.56 & 7.27 & 160.45 & 1.47 & 418.87 \\
\hline PCUCP-6 & 40.80 & 5.07 & 61.33 & 3.20 & 15.37 & 4.28 & 10.33 & 125.93 & 1.58 & 417.86 \\
\hline PCUCP-7 & 37.89 & 6.07 & 62.00 & 3.00 & 16.55 & 4.19 & 8.40 & 177.90 & 1.96 & 517.51 \\
\hline PCUCP-8 & 39.33 & 3.60 & 58.00 & 3.07 & 16.50 & 3.84 & 16.20 & 132.41 & 3.04 & 801.33 \\
\hline PGYC-1 & 33.87 & 10.20 & 70.00 & 4.02 & 12.36 & 3.97 & 10.07 & 120.84 & 1.60 & 421.15 \\
\hline PGYC-2 & 40.87 & 5.20 & 77.00 & 4.88 & 15.30 & 4.09 & 7.13 & 170.68 & 1.69 & 445.60 \\
\hline PGYC-3 & 40.00 & 4.93 & 72.67 & 5.62 & 13.72 & 4.58 & 11.40 & 168.66 & 2.45 & 646.52 \\
\hline PCUC-8 & 49.20 & 24.00 & 63.67 & 4.85 & 16.50 & 4.44 & 2.27 & 195.00 & 0.48 & 126.35 \\
\hline
\end{tabular}




\begin{tabular}{|c|c|c|c|c|c|c|c|c|c|c|}
\hline PCUC-25 & 56.20 & 26.93 & 67.33 & 4.50 & 18.50 & 4.24 & 2.07 & 188.00 & 0.37 & 105.24 \\
\hline Pant Khira-1 & 49.13 & 27.00 & 72.00 & 5.78 & 19.00 & 4.20 & 2.20 & 200.00 & 0.50 & 130.48 \\
\hline PCUCP-1×PCUC-8 & 39.07 & 3.07 & 56.33 & 6.07 & 15.00 & 3.53 & 16.47 & 133.82 & 2.79 & 736.22 \\
\hline PCUCP-1×PCUC-25 & 37.87 & 9.00 & 58.22 & 3.82 & 16.80 & 3.86 & 16.40 & 220.79 & 4.33 & 1140.70 \\
\hline PCUCP-1×Pant Khira-1 & 36.87 & 8.13 & 57.33 & 5.20 & 16.24 & 3.40 & 15.80 & 192.87 & 4.55 & 1201.45 \\
\hline PCUCP-2×PCUC-8 & 38.00 & 4.07 & 56.67 & 6.51 & 16.39 & 3.60 & 12.27 & 199.33 & 3.23 & 851.72 \\
\hline PCUCP-2×PCUC-25 & 36.80 & 10.20 & 54.00 & 7.00 & 14.50 & 3.50 & 16.27 & 135.47 & 2.88 & 759.28 \\
\hline PCUCP-2×Pant Khira-1 & 39.00 & 2.07 & 55.00 & 6.50 & 15.60 & 4.48 & 13.60 & 169.53 & 3.40 & 899.45 \\
\hline PCUCP-3×PCUC-8 & 40.33 & 8.27 & 57.67 & 5.90 & 20.24 & 4.24 & 11.47 & 259.70 & 4.35 & 1144.94 \\
\hline PCUCP-3×PCUC-25 & 40.33 & 6.07 & 61.00 & 3.50 & 16.63 & 3.26 & 16.00 & 270.33 & 5.19 & 1367.44 \\
\hline PCUCP-3×Pant Khira-1 & 40.53 & 10.27 & 58.33 & 4.80 & 14.63 & 3.24 & 8.40 & 164.27 & 2.07 & 545.67 \\
\hline PCUCP-4×PCUC-8 & 37.87 & 14.00 & 57.67 & 4.31 & 17.64 & 3.87 & 19.00 & 197.25 & 4.67 & 1235.36 \\
\hline PCUCP-4×PCUC-25 & 35.93 & 6.80 & 54.33 & 4.36 & 14.17 & 4.00 & 15.07 & 144.65 & 3.05 & 798.77 \\
\hline PCUCP-4×Pant Khira-1 & 40.00 & 2.93 & 54.67 & 3.81 & 16.19 & 4.80 & 12.07 & 155.15 & 2.79 & 736.40 \\
\hline PCUCP-5×PCUC-8 & 36.87 & 4.00 & 57.67 & 6.00 & 16.33 & 3.77 & 11.80 & 210.54 & 3.77 & 993.69 \\
\hline PCUCP-5×PCUC-25 & 39.47 & 6.73 & 61.67 & 5.03 & 14.00 & 3.71 & 10.00 & 160.62 & 2.20 & 579.61 \\
\hline PCUCP-5×Pant Khira-1 & 44.60 & 11.93 & 59.00 & 4.35 & 13.70 & 3.77 & 11.00 & 200.00 & 3.01 & 795.19 \\
\hline PCUCP-6×PCUC-8 & 41.80 & 7.93 & 61.67 & 5.07 & 13.64 & 4.44 & 8.80 & 150.00 & 1.91 & 504.19 \\
\hline PCUCP-6×PCUC-25 & 38.00 & 8.27 & 58.00 & 5.48 & 14.60 & 3.89 & 10.20 & 215.21 & 3.00 & 792.92 \\
\hline PCUCP-6×Pant Khira-1 & 39.80 & 9.00 & 58.00 & 4.85 & 18.80 & 3.89 & 9.00 & 226.27 & 2.71 & 713.74 \\
\hline PCUCP-7×PCUC-8 & 45.07 & 9.73 & 62.00 & 4.93 & 16.57 & 3.55 & 10.20 & 172.65 & 2.33 & 615.35 \\
\hline PCUCP-7×PCUC-25 & 39.00 & 11.00 & 58.00 & 5.65 & 18.85 & 4.20 & 11.20 & 159.94 & 2.69 & 709.13 \\
\hline PCUCP-7×Pant Khira-1 & 45.00 & 14.00 & 62.33 & 5.10 & 17.00 & 3.47 & 6.80 & 116.08 & 1.05 & 278.16 \\
\hline PCUCP-8×PCUC-8 & 38.33 & 6.80 & 57.67 & 4.76 & 17.00 & 3.66 & 7.13 & 170.92 & 1.84 & 485.30 \\
\hline PCUCP-8×PCUC-25 & 38.33 & 4.00 & 61.67 & 5.25 & 15.33 & 4.09 & 9.20 & 150.43 & 1.82 & 480.44 \\
\hline PCUCP-8×Pant Khira-1 & 40.20 & 7.07 & 61.67 & 5.10 & 18.61 & 3.74 & 5.80 & 220.60 & 1.86 & 488.17 \\
\hline PGYC-1×PCUC-8 & 41.33 & 4.00 & 57.67 & 4.87 & 13.61 & 3.88 & 8.20 & 160.71 & 1.71 & 449.06 \\
\hline PGYC-1×PCUC-25 & 38.00 & 4.93 & 62.00 & 5.60 & 13.66 & 4.04 & 8.00 & 170.00 & 1.69 & 448.28 \\
\hline PGYC-1×Pant Khira-1 & 47.87 & 6.93 & 62.00 & 4.02 & 14.11 & 4.16 & 8.20 & 175.00 & 1.89 & 500.58 \\
\hline PGYC-2×PCUC-8 & 60.47 & 9.07 & 71.00 & 5.00 & 19.80 & 4.00 & 6.67 & 170.00 & 1.56 & 412.28 \\
\hline PGYC-2×PCUC-25 & 48.00 & 7.13 & 77.00 & 6.03 & 21.34 & 4.30 & 8.00 & 175.00 & 1.88 & 497.66 \\
\hline PGYC-2×Pant Khira-1 & 40.33 & 18.27 & 77.00 & 6.10 & 21.60 & 4.40 & 8.53 & 178.00 & 2.01 & 535.23 \\
\hline PGYC-3×PCUC-8 & 60.47 & 9.27 & 70.00 & 6.25 & 13.56 & 5.26 & 7.00 & 160.00 & 1.52 & 401.30 \\
\hline PGYC-3×PCUC-25 & 40.40 & 5.27 & 62.00 & 5.20 & 12.30 & 4.27 & 7.13 & 200.00 & 1.95 & 514.96 \\
\hline PGYC-3×Pant Khira-1 & 40.00 & 20.20 & 85.00 & 6.03 & 14.00 & 5.34 & 8.20 & 210.00 & 2.12 & 560.44 \\
\hline Mean & 41.55 & 8.66 & 62.21 & 4.84 & 16.06 & 4.04 & 10.23 & 176.72 & 2.42 & 638.86 \\
\hline C.V. & 1.32 & 5.31 & 1.44 & 8.00 & 0.61 & 1.09 & 9.09 & 6.41 & 8.61 & 3.36 \\
\hline S.E. & 0.32 & 0.27 & 0.52 & 0.22 & 0.06 & 0.03 & 0.54 & 6.54 & 0.12 & 12.40 \\
\hline C.D. $5 \%$ & 0.89 & 0.75 & 1.46 & 0.63 & 0.16 & 0.07 & 1.51 & 18.36 & 0.34 & 34.82 \\
\hline C.D. $1 \%$ & 1.18 & 0.99 & 1.93 & 0.83 & 0.21 & 0.09 & 2.00 & 24.31 & 0.45 & 46.11 \\
\hline
\end{tabular}

Table 3: Heterosis over better parent for different traits in cucumber in E1

\begin{tabular}{|c|c|c|c|c|c|c|c|c|c|c|}
\hline Genotypes & DFFFA & NNFFF & DFFH & VL (m) & FL (cm) & FD $(\mathbf{c m})$ & NF/P & AFW (g) & FY/P (kg) & FY/Ha(q) \\
\hline PCUCP-1×PCUC-8 & $-10.55 * *$ & $-17.72 *$ & $-9.57 * *$ & $142.56 * *$ & 1.27 & $6.86 *$ & $-30.00 * *$ & 8.91 & $-19.93 * *$ & $-19.97 * *$ \\
\hline PCUCP-1×PCUC-25 & $14.41 * *$ & $113.92 * *$ & $3.72 * *$ & $46.84 * *$ & $8.51 * *$ & $11.96 * *$ & -8.67 & $47.32 * *$ & $46.38 * *$ & $46.10 * *$ \\
\hline PCUCP-1×Pant Khira-1 & $3.57 * *$ & $37.97 * *$ & $4.79 * *$ & $102.56^{* *}$ & $5.57 * *$ & -1.76 & -1.33 & $29.14 * *$ & $35.51 * *$ & $35.05 * *$ \\
\hline PCUCP-2×PCUC-8 & $-19.45 * *$ & -4.76 & $-7.69 * *$ & $21.14 * *$ & $4.51 * *$ & -2.73 & $-29.51 * *$ & 9.32 & $-25.77 * *$ & $-25.80 * *$ \\
\hline PCUCP-2×PCUC-25 & $-11.64 * *$ & $23.81 *$ & $-4.95 * *$ & -6.10 & $-6.27 * *$ & -1.82 & $-28.42 * *$ & 0.26 & $-22.55 * *$ & $-22.65 * *$ \\
\hline PCUCP-2×Pant Khira-1 & $5.62 * *$ & $165.08 * *$ & $11.54 * *$ & $-27.56 * *$ & 0.56 & $27.82 * *$ & $-34.97 * *$ & $17.16 * *$ & $-26.33 * *$ & $-26.53 * *$ \\
\hline PCUCP-3×PCUC-8 & $4.40^{* *}$ & $25.81 * *$ & 1.70 & $43.57 * *$ & $2.41 * *$ & $18.60 * *$ & -0.72 & $55.20 * *$ & $60.88 * *$ & $61.43 * *$ \\
\hline PCUCP-3×PCUC-25 & $-7.24 * *$ & -1.08 & -1.70 & $22.14 *$ & $-15.78 * *$ & $-14.74 * *$ & -11.59 & 7.89 & -1.59 & -1.46 \\
\hline PCUCP-3×Pant Khira-1 & $2.27 * *$ & $68.82 * *$ & 0.57 & $68.14 * *$ & $-25.87 * *$ & $-15.79 * *$ & $-31.88 * *$ & 3.94 & $-22.83 * *$ & $-22.62 * *$ \\
\hline PCUCP-4×PCUC-8 & $15.00^{* *}$ & 15.25 & $3.45 * *$ & $58.43 * *$ & $41.98^{* *}$ & $-19.41 * *$ & $65.69 * *$ & 0.42 & $55.71 * *$ & $56.10^{* *}$ \\
\hline PCUCP-4×PCUC-25 & $2.67 *$ & $23.73^{*}$ & $-10.34 * *$ & $39.42 * *$ & $15.07 * *$ & $-12.52 * *$ & 18.63 & -6.02 & 9.80 & $10.49^{* *}$ \\
\hline PCUCP-4×Pant Khira-1 & $-7.50 * *$ & $27.12 *$ & $-2.87 *$ & $38.15 * *$ & $31.98 * *$ & 1.27 & -5.88 & $21.24 * *$ & $20.82 *$ & $21.22 * *$ \\
\hline PCUCP-5×PCUC-8 & $19.07 * *$ & 20.00 & $-9.76 * *$ & $37.81 * *$ & $31.61 * *$ & $-17.26 * *$ & $97.22 * *$ & 7.98 & $152.88 * *$ & $154.17 * *$ \\
\hline PCUCP-5×PCUC-25 & $19.26^{* *}$ & $50.00 * *$ & $-3.90 * *$ & $-21.88 * *$ & $9.22 * *$ & $-18.14 * *$ & $68.06 * *$ & 0.37 & $58.99 * *$ & $59.23 * *$ \\
\hline PCUCP-5×Pant Khira-1 & $19.63^{* *}$ & $48.33^{* *}$ & $-21.95 * *$ & $-35.94 * *$ & $10.36 * *$ & $-17.26 * *$ & $33.33 *$ & $26.52 * *$ & $83.81 * *$ & $84.70 * *$ \\
\hline PCUCP-6×PCUC-8 & $7.59 * *$ & $42.19 * *$ & $-4.92 * *$ & $59.84 * *$ & $-11.15^{* *}$ & 3.94 & 17.24 & $29.22 * *$ & $89.47 * *$ & $89.55 * *$ \\
\hline PCUCP-6×PCUC-25 & $9.44 * *$ & $89.06 * *$ & $-4.92 * *$ & $36.39 * *$ & $-4.72 * *$ & $8.11 * *$ & $29.89 *$ & $82.05 * *$ & $170.61 * *$ & $174.99 * *$ \\
\hline PCUCP-6×Pant Khira-1 & $5.57 * *$ & $93.75^{* *}$ & $-5.46^{* *}$ & $86.07 * *$ & $22.97 * *$ & $6.30 * *$ & $29.89^{*}$ & $81.99 * *$ & 11.84 & $159.76 * *$ \\
\hline PCUCP-7×PCUC-8 & $5.58 * *$ & $294.12 * *$ & 0.64 & $78.00 * *$ & $8.12 * *$ & $-14.81 * *$ & $30.91 * *$ & -0.66 & $37.84 * *$ & $35.62 * *$ \\
\hline PCUCP-7×PCUC-25 & $26.44 * *$ & $379.41 * *$ & $19.23 * *$ & $113.69 * *$ & $24.00 * *$ & 3.70 & -15.45 & $25.31 * *$ & 15.69 & $15.88^{* *}$ \\
\hline PCUCP-7×Pant Khira-1 & $33.00 * *$ & $494.12 * *$ & $14.10 * *$ & $84.62 * *$ & $7.98 * *$ & $-17.23 * *$ & -15.45 & $-40.17 * *$ & 9.22 & $-47.77 * *$ \\
\hline PCUCP-8×PCUC-8 & $2.37 *$ & $110.20 * *$ & $-7.10 * *$ & $85.00 * *$ & $45.67 * *$ & -3.86 & $-46.32 * *$ & $38.43 * *$ & $-30.00 * *$ & $-13.73 * *$ \\
\hline PCUCP-8×PCUC-25 & $-13.20 * *$ & 22.45 & 1.78 & $113.67 * *$ & $27.11 * *$ & $5.61 *$ & $-36.84 * *$ & 10.71 & $-37.56 * *$ & $-23.05 * *$ \\
\hline PCUCP-8×Pant Khira-1 & 1.19 & $83.67 * *$ & $7.69 * *$ & $90.00 * *$ & $54.56 * *$ & -3.33 & $-67.37 * *$ & $31.01 * *$ & $-62.69 * *$ & $-53.55 * *$ \\
\hline PGYC-1×PCUC-8 & $8.70 * *$ & 17.65 & $-21.11 * *$ & $68.75 * *$ & $11.09 * *$ & -2.95 & -11.11 & $79.31 * *$ & $79.61 * *$ & $80.88 * *$ \\
\hline
\end{tabular}




\begin{tabular}{|c|c|c|c|c|c|c|c|c|c|c|}
\hline PGYC-1×PCUC-25 & $4.53 * *$ & $47.06^{* *}$ & $-14.07 * *$ & $60.00 * *$ & $11.04 * *$ & 1.27 & 12.22 & $84.01 * *$ & $77.67 * *$ & $80.83 * *$ \\
\hline PGYC-1×Pant Khira-1 & $9.60 * *$ & $105.88 * *$ & $-21.61 * *$ & $43.12 * *$ & $14.75 * *$ & 3.80 & 13.33 & $100.32 * *$ & $139.32 * *$ & $141.45^{* *}$ \\
\hline PGYC-2×PCUC-8 & $10.42 * *$ & $91.25 * *$ & $28.57 * *$ & $-15.32 * *$ & $28.01 * *$ & -0.66 & -12.90 & 0.18 & -6.85 & -6.42 \\
\hline PGYC-2×PCUC-25 & $11.07 * *$ & $31.25 * *$ & $10.29 * *$ & $-15.25 * *$ & $39.30 * *$ & $5.63 *$ & 0.00 & 2.41 & 15.89 & $16.43 * *$ \\
\hline PGYC- $2 \times$ Pant Khira- 1 & $67.26^{* *}$ & $243.75 * *$ & $32.57 * *$ & $-22.03 * *$ & $39.93 * *$ & $8.28 * *$ & $29.03^{* *}$ & 0.82 & $30.56 * *$ & $30.59 * *$ \\
\hline PGYC-3×PCUC-8 & $12.13^{* *}$ & $11.38 *$ & $-3.45^{* *}$ & $50.73 * *$ & -1.51 & $15.32 * *$ & -17.65 & -7.44 & -8.70 & $-8.87 *$ \\
\hline PGYC-3×PCUC-25 & $35.05^{* *}$ & $-53.66 * *$ & $5.91 * *$ & $11.83^{*}$ & $-10.31 * *$ & $-6.63^{* * *}$ & -11.76 & $21.24 * *$ & 13.91 & $14.20^{* * *}$ \\
\hline PGYC-3×Pant Khira-1 & $51.99 * *$ & $129.27 * *$ & $3.94 * *$ & $13.05^{*}$ & $2.59 * *$ & $17.23 * *$ & -8.82 & $29.49 * *$ & 16.30 & $16.68^{* * *}$ \\
\hline
\end{tabular}

NNFFF $=$ Node No. of first female flower, DFFFA $=$ Days to first female flower anthesis, DFFH = Days to first fruit harvest, VL $=$ Vine Length, FL = Fruit length, FD = Fruit diameter, NF/P = No. of fruits per plant, AFW = Average fruit weight, FY/P = Yield per plant, FY/ Ha= Yield per hectare, PCUCP-2=Pant parthenocarpic Cucumber-2, PCUCP-3=Pant parthenocarpic Cucumber-3

Table 4: Heterosis over better parent for different traits in cucumber in E2

\begin{tabular}{|c|c|c|c|c|c|c|c|c|c|c|}
\hline Genotypes & DFFFA & NNFFF & DFFH & VL (m) & FL (cm) & FD $(\mathbf{c m})$ & $\mathbf{N F} / \mathbf{P}$ & AFW (g) & FY/P (kg) & FY/Ha (q) \\
\hline PCUCP-1×PCUC- 8 & $-17.00 * *$ & $-41.77 * *$ & -2.31 & $213.79 * *$ & $20.00 * *$ & 0.95 & $37.22 * *$ & -4.41 & $34.57 * *$ & $34.21 * *$ \\
\hline PCUCP-1×PCUC-25 & $-19.55 * *$ & $70.89 * *$ & 0.96 & $97.59 * *$ & $34.40^{* *}$ & $10.29^{* *}$ & $36.67 * *$ & $57.71 * *$ & 108.84** & $107.95^{* *}$ \\
\hline PCUCP-1×Pant Khira-1 & $-21.67 * *$ & $54.43^{* *}$ & -0.58 & $168.97 * *$ & $29.92^{* *}$ & $-2.86^{* *}$ & $31.67^{* * *}$ & $37.76^{* *}$ & $119.45^{* *}$ & $119.02 * *$ \\
\hline PCUCP-2×PCUC-8 & $26.67 * *$ & -4.69 & -2.30 & $54.43 * *$ & $-9.23 * *$ & $-2.70^{* *}$ & $-17.12 * *$ & $15.70 * *$ & 1.79 & 2.28 \\
\hline PCUCP-2×PCUC-25 & $22.67 * *$ & $139.06^{* *}$ & $-6.90 * *$ & $66.14 * *$ & $-19.68 * *$ & $-5.41 * *$ & 9.91 & $-21.37 * *$ & -9.14 & $-8.82 * *$ \\
\hline PCUCP-2×Pant Khira-1 & $30.00 * *$ & $-51.56^{* *}$ & $-5.17 * *$ & $54.27 * *$ & $-13.59 * *$ & $21.08^{* *}$ & -8.11 & -1.60 & 7.25 & $8.01 * *$ \\
\hline PCUCP-3×PCUC-8 & $12.24 * *$ & $37.78 * *$ & $-3.89 * *$ & $84.37 * *$ & $-9.48 * *$ & $10.42 * *$ & -7.53 & $41.44 * *$ & $43.93 * *$ & $44.76 * *$ \\
\hline PCUCP-3×PCUC- 25 & $12.24 * *$ & 1.11 & 1.67 & 9.37 & $-25.61 * *$ & $-15.10^{* *}$ & $29.03^{* *}$ & $47.23^{* *}$ & $71.74 * *$ & $72.89 * *$ \\
\hline PCUCP-3×Pant Khira- 1 & $12.80 * *$ & $71.11 * *$ & $-2.78^{*}$ & $50.00^{* * *}$ & $-34.59 * *$ & $-15.62 * *$ & $-32.26 * *$ & $-10.53 *$ & $-31.57 * *$ & $-31.01 * *$ \\
\hline PCUCP-4×PCUC-8 & $-17.92 * *$ & $180.00 * *$ & 0.00 & $58.65^{* *}$ & $35.48^{* *}$ & $-17.73 * *$ & $79.25^{* *}$ & $12.69^{*}$ & $89.97 * *$ & $90.03 * *$ \\
\hline PCUCP-4×PCUC-25 & $-22.11 * *$ & $36.00 * *$ & $-5.78 * *$ & $60.61 * *$ & $8.86 * *$ & $-14.89 * *$ & $42.14 * *$ & $-17.36^{* *}$ & $24.12^{* *}$ & $22.87 * *$ \\
\hline PCUCP-4×Pant Khira-1 & $-13.29 * *$ & $-41.33 * *$ & $-5.20 * *$ & $40.12 * *$ & $24.37 * *$ & $2.13 * *$ & 13.84 & $-11.37 *$ & 13.55 & $13.28 * *$ \\
\hline PCUCP-5×PCUC-8 & $-8.60 * *$ & $36.36^{* * *}$ & $-7.49 * *$ & $50.00^{* * *}$ & $31.31 * *$ & $-17.25 * *$ & $62.39 * *$ & $31.22 * *$ & $156.24 * *$ & $137.23 * *$ \\
\hline PCUCP-5×PCUC-25 & -2.15 & $129.55 * *$ & -1.07 & $25.83^{* *}$ & $12.60^{* *}$ & $-18.71 * *$ & $37.61^{* *}$ & 0.11 & $49.43 * *$ & $38.38 * *$ \\
\hline PCUCP-5×Pant Khira-1 & $10.58 * *$ & $306.82 * *$ & $-5.35 * *$ & 8.75 & $10.19^{* *}$ & $-17.25^{* *}$ & $51.38^{* *}$ & $24.65 * *$ & $104.76 * *$ & $89.84 * *$ \\
\hline PCUCP-6×PCUC-8 & $2.45^{*}$ & $56.58 * *$ & 0.54 & $58.33^{* * *}$ & $-11.27 * *$ & $3.74 * *$ & $-14.84 *$ & $19.12 *$ & 20.89 & $20.66 * *$ \\
\hline PCUCP-6×PCUC-25 & -6.86 & $63.16^{* *}$ & $-5.43 * *$ & $71.35^{* *}$ & $-5.03 * *$ & $-9.19 * *$ & -1.29 & $70.90 * *$ & $89.87 * *$ & $89.76^{* *}$ \\
\hline PCUCP-6×Pant Khira-1 & -2.45 & $77.63 * *$ & $-5.43 * *$ & $51.46^{* *}$ & $22.29 * *$ & $-9.19 * *$ & -12.90 & $79.69 * *$ & $71.31 * *$ & $70.81 * *$ \\
\hline PCUCP-7×PCUC-8 & 18.95 & $60.44 * *$ & 0.00 & $64.44 * *$ & 0.08 & $-15.13 * *$ & $21.43^{*}$ & -2.95 & $19.05^{*}$ & $18.91 * *$ \\
\hline PCUCP-7×PCUC-25 & 2.94 & $81.32 * *$ & $-6.45 * *$ & $88.33^{* *}$ & $13.85^{* *}$ & 0.32 & $33.33^{* *}$ & -10.10 & $37.24 * *$ & $37.03 * *$ \\
\hline PCUCP-7×Pant Khira-1 & 18.78 & $130.77 * *$ & 0.54 & $70.00^{* *}$ & $2.70 * *$ & $-17.04 * *$ & $-19.05^{*}$ & $-34.75 * *$ & $-46.26 * *$ & $-46.25 * *$ \\
\hline PCUCP-8×PCUC-8 & -2.54 & $88.89 * *$ & -0.57 & $55.22 * *$ & $3.03 * *$ & $-4.69 * *$ & $-55.97 * *$ & $29.08 * *$ & $-39.58 * *$ & $-39.44 * *$ \\
\hline PCUCP- $8 \times$ PCUC- 25 & -2.54 & 11.11 & $6.32 * *$ & $71.20^{* * *}$ & $-7.11 * *$ & $6.42 * *$ & -43.21 & 13.61 & -40.24 & $-40.04 * *$ \\
\hline PCUCP-8×Pant Khira-1 & 2.20 & $96.30 * *$ & $6.32 * *$ & $66.30^{* * *}$ & $12.81^{* *}$ & $-2.60 * *$ & $-64.20 * *$ & $66.61 * *$ & $-38.93 * *$ & $-39.08 * *$ \\
\hline PGYC-1×PCUC-8 & 22.05 & $-60.78^{* *}$ & $-17.62 * *$ & $21.16^{* *}$ & $10.14^{* *}$ & $-2.18^{*}$ & $-18.54 *$ & $32.99^{* *}$ & 6.87 & 6.63 \\
\hline PGYC-1×PCUC-25 & 12.20 & $-51.63 * *$ & $-11.43 * *$ & $39.42 * *$ & $10.52^{* *}$ & $30.32 * *$ & $-20.53 * *$ & $40.68^{* *}$ & 5.62 & 6.44 \\
\hline PGYC-1×Pant Khira-1 & 41.34 & $-32.03 * *$ & $-11.43^{* *}$ & 0.00 & $14.13^{* *}$ & $34.09^{* *}$ & $-18.54 *$ & $44.82 * *$ & 18.12 & $18.86 * *$ \\
\hline PGYC-2×PCUC-8 & 47.96 & $74.36 * *$ & $-7.79 * *$ & 2.39 & $29.41^{* *}$ & $-2.12 *$ & -6.54 & -0.40 & -7.51 & $-7.48 * *$ \\
\hline PGYC-2×PCUC-25 & 17.46 & $37.18 * *$ & 0.00 & $23.55^{* * *}$ & $39.48^{* *}$ & $5.22 * *$ & 12.15 & 2.53 & 11.46 & $11.68 * *$ \\
\hline PGYC-2×Pant Khira-1 & -1.31 & $251.28 * *$ & 0.00 & $24.91 * *$ & $41.18^{* *}$ & $7.67 * *$ & 19.63 & 4.29 & 18.97 & $20.11 * *$ \\
\hline PGYC-3×PCUC-8 & 51.17 & $87.84 * *$ & $-3.67 * *$ & $11.28^{*}$ & $-1.17 *$ & $14.77^{* * *}$ & $-38.60 * *$ & -5.13 & $-37.87 * *$ & $-37.93 * *$ \\
\hline PGYC-3×PCUC-25 & 1.00 & 6.76 & $-14.68 * *$ & -7.42 & $-10.35 * *$ & $-6.70^{* * *}$ & $-37.43 * *$ & $18.58 * *$ & $-20.30 * *$ & $-20.35 * *$ \\
\hline PGYC-3×Pant Khira-1 & 0.00 & $309.46^{* *}$ & $16.97 * *$ & 7.42 & $2.04 * *$ & $16.59^{* * *}$ & $-28.07 * *$ & $24.51 * *$ & -13.35 & $-13.31 * *$ \\
\hline
\end{tabular}

NNFFF = Node No. of first female flower, DFFFA = Days to first female flower anthesis, DFFH = Days to first fruit harvest, VL = Vine Length, FL = Fruit length, $\mathrm{FD}=$ Fruit diameter, NF/P $=$ No. of fruits per plant, $\mathrm{AFW}=$ Average fruit weight, $\mathrm{FY} / \mathrm{P}=\mathrm{Yield}$ per plant, $\mathrm{FY} / \mathrm{Ha}=\mathrm{Yield}$ per hectare, $\mathrm{PCUCP}-2=\mathrm{Pant}$ parthenocarpic Cucumber-2, PCUCP-3=Pant parthenocarpic Cucumber-3

Table 5: Heterosis over standard parent for different traits in cucumber E1

\begin{tabular}{|c|c|c|c|c|c|c|c|c|c|c|}
\hline Character & DFFFA & NNFFF & DFFH & \begin{tabular}{|l|}
$\mathrm{VL}(\mathrm{m})$ \\
\end{tabular} & FL (cm) & FD (cm) & $\mathbf{N F} / \mathbf{P}$ & AFW (g) & FY/P (kg) & FY/Ha (q) \\
\hline PCUCP-1×PCUC- 8 & $-44.16^{* *}$ & $-75.38 * *$ & $-30.61 * *$ & $20.56^{* *}$ & $-17.65^{* *}$ & $-11.38^{* *}$ & $250.00 * *$ & $-21.59 * *$ & $220.29 * *$ & $220.26 * *$ \\
\hline PCUCP-1×PCUC-25 & $-28.57 * *$ & $-35.98 * *$ & $-20.41 * *$ & $-27.02 * *$ & $-11.76 * *$ & $-7.15 * *$ & $356.67 * *$ & 6.07 & $485.51 * *$ & $484.62 * *$ \\
\hline PCUCP-1×Pant Khira-1 & $-35.34 * *$ & $-58.71 * *$ & $-19.59 * *$ & 0.68 & $-14.15 * *$ & $-18.54 * *$ & $393.33^{* *}$ & -7.02 & $442.03 * *$ & $440.40 * *$ \\
\hline PCUCP-2×PCUC-25 & $-40.17 * *$ & $-70.45 * *$ & $-29.39 * *$ & -1.87 & $-22.21 * *$ & $-12.20 * *$ & $336.67 * *$ & $-25.39 * *$ & $300.72 * *$ & $300.47 * *$ \\
\hline PCUCP-2×Pant Khira-1 & $-28.48 * *$ & $-36.74 * *$ & $-17.14 * *$ & $-24.30 * *$ & $-16.54 * *$ & $14.31 * *$ & $296.67 * *$ & $-12.81 * *$ & $281.16 * *$ & $280.41 * *$ \\
\hline PCUCP-3×PCUC-8 & $-31.82 * *$ & $-55.68 * *$ & $-26.94 * *$ & $-14.61 *$ & $7.56^{* * *}$ & $9.92 * *$ & $356.67 * *$ & $20.08 * *$ & $558.70 * *$ & $562.21 * *$ \\
\hline PCUCP-3×PCUC-25 & $-39.42 * *$ & $-65.15 * *$ & $-29.39 * *$ & $-27.36^{* *}$ & $-11.55 * *$ & $-20.98 * *$ & $306.67 * *$ & $-16.52 * *$ & $302.90 * *$ & $304.22 * *$ \\
\hline PCUCP-3×Pant Khira-1 & $-33.21 * *$ & $-40.53 * *$ & $-27.76 * *$ & 0.00 & $-22.14 * *$ & $-21.95 * *$ & $213.33 * *$ & $-19.58 * *$ & $215.94 * *$ & $217.45 * *$ \\
\hline PCUCP-4×PCUC-8 & $-35.99 * *$ & $-74.24 * *$ & $-26.53 * *$ & 6.20 & $-6.31 * *$ & $-6.83^{* *}$ & $463.33^{* *}$ & $-11.88 * *$ & $452.90 * *$ & $452.57 * *$ \\
\hline PCUCP-4×PCUC-25 & $-42.86^{* * *}$ & $-72.35 * *$ & $-36.33 * *$ & -6.54 & $-24.06 * *$ & 1.14 & $303.33 * *$ & $-17.52 * *$ & $289.86 * *$ & $291.12 * *$ \\
\hline PCUCP-4×Pant Khira-1 & $-48.52 * *$ & $-71.59 * *$ & $-31.02 * *$ & -7.39 & $-12.91 * *$ & $17.07 * *$ & $220.00 * *$ & 6.40 & $328.99 * *$ & $329.08 * *$ \\
\hline PCUCP-5×PCUC-8 & $-40.35 * *$ & $-72.73 * *$ & $-24.49 * *$ & $12.40 *$ & $-13.01 * *$ & $-8.78 * *$ & $373.33 * *$ & $-18.86^{* *}$ & $409.42 * *$ & $409.65 * *$ \\
\hline PCUCP-5×PCUC-25 & $-40.26 * *$ & $-65.91 * *$ & $-19.59 * *$ & $-36.28 * *$ & $-27.81 * *$ & $-9.76^{* *}$ & $303.33 * *$ & $-24.58 * *$ & $220.29 * *$ & $219.27 * *$ \\
\hline PCUCP-5×Pant Khira-1 & $-40.07 * *$ & $-66.29 * *$ & $-34.69 * *$ & $-47.75 * *$ & $-27.06^{* *} *$ & $-8.78 * *$ & $220.00 * *$ & -4.94 & $270.29 * *$ & $270.34 * *$ \\
\hline PCUCP-6×PCUC-8 & $-35.53 * *$ & $-65.53 * *$ & $-28.98 * *$ & $-17.16^{* *}$ & $-27.59 * *$ & $7.32^{* *}$ & $240.00 * *$ & $-28.76 * *$ & $213.04 * *$ & $212.75 * *$ \\
\hline PCUCP-6×Pant Khira-1 & $-36.73 * *$ & $-53.03 * *$ & $-29.39 * *$ & -3.57 & 0.21 & $9.76^{* * *}$ & $276.67 * *$ & \begin{tabular}{|l|}
0.33 \\
\end{tabular} & $84.78 * *$ & $328.60 * *$ \\
\hline PCUCP-7×PCUC-8 & $-40.35 * *$ & $-49.24 * *$ & $-35.92 * *$ & -1.70 & $-11.66^{* *}$ & $-13.98 * *$ & $380.00 * *$ & $-15.09 * *$ & $409.42 * *$ & $400.10^{* *}$ \\
\hline PCUCP-7×PCUC-25 & $-28.57 * *$ & $-38.26 * *$ & $-24.08 * *$ & $18.01 * *$ & $1.32 *$ & $4.72 *$ & $210.00 * *$ & 7.10 & $327.54 * *$ & $327.32 * *$ \\
\hline
\end{tabular}




\begin{tabular}{|c|c|c|c|c|c|c|c|c|c|c|}
\hline PCUCP-7×Pant Khira-1 & $-24.86^{* *}$ & $-23.48 * *$ & $-27.35 * *$ & 1.95 & $-11.76 * *$ & $-16.42 * *$ & $210.00 * *$ & $-48.86 * *$ & $303.62 * *$ & $92.59 * *$ \\
\hline PCUCP-8×PCUC-8 & $-35.99 * *$ & $-60.98 * *$ & $-35.92 * *$ & -5.69 & $-6.52 * *$ & $-10.89 * *$ & $240.00 * *$ & $-10.02 *$ & $295.65 * *$ & $295.38 * *$ \\
\hline PCUCP-8×PCUC-25 & $-45.73 * *$ & $-77.27 * *$ & $-29.80 * *$ & 8.92 & $-18.43 * *$ & -2.11 & $300.00 * *$ & $-28.04 * *$ & $252.90 * *$ & $252.69 * *$ \\
\hline PCUCP-8×Pant Khira-1 & $-36.73 * *$ & $-65.91 * *$ & $-25.71 * *$ & -3.14 & -0.82 & $-10.41 * *$ & $106.67 * *$ & $-14.84 * *$ & $110.87 * *$ & $112.90 * *$ \\
\hline PGYC-1×PCUC-8 & $-44.34 * *$ & $-77.27 * *$ & $-35.92 * *$ & $14.70^{*}$ & $-27.52 * *$ & $-6.50 * *$ & $166.67 * *$ & $-23.80 * *$ & $168.12 * *$ & $167.24 * *$ \\
\hline PGYC-1×PCUC-25 & $-46.47 * *$ & $-71.59 * *$ & $-30.20 * *$ & 8.75 & $-27.56 * *$ & \begin{tabular}{|l|}
-2.44 \\
\end{tabular} & $236.67 * *$ & $-21.80 * *$ & $165.22 * *$ & $167.16^{* *}$ \\
\hline PGYC-1×Pant Khira-1 & $-43.88 * *$ & $-60.23 * *$ & $-36.33 * *$ & -2.72 & $-25.13 * *$ & 0.00 & $240.00 * *$ & $-14.87 * *$ & $257.25 * *$ & $256.73 * *$ \\
\hline PGYC-2×PCUC-8 & $-37.11 * *$ & $-42.05 * *$ & $-8.16 * *$ & 6.12 & $4.28 * *$ & -2.44 & $170.00 * *$ & $-15.54 * *$ & $176.09 * *$ & $177.49 * *$ \\
\hline PGYC-2×PCUC-25 & $-36.73 * *$ & $-60.23 * *$ & $-21.22 * *$ & 6.20 & $13.48 * *$ & 3.74 & $210.00 * *$ & $-13.66 * *$ & $243.48 * *$ & $245.26 * *$ \\
\hline PGYC-2×Pant Khira-1 & $-4.73 * *$ & $4.17 * *$ & $-5.31 * *$ & -2.29 & $13.99 * *$ & $6.34 * *$ & $300.00 * *$ & $-15.00 * *$ & $286.96 * *$ & $287.25 * *$ \\
\hline PGYC-3×PCUC-8 & $-37.38 * *$ & $-48.11 * *$ & $-20.00 * *$ & $58.03 * *$ & $-28.13 * *$ & $27.32 * *$ & $180.00 * *$ & $-24.83 * *$ & $204.35 * *$ & $202.61 * *$ \\
\hline PGYC-3×PCUC-25 & $-24.58 * *$ & $-78.41^{* *}$ & $-12.24 * *$ & $17.25 * *$ & $-34.55 * *$ & 3.09 & $200.00 * *$ & -1.53 & $279.71 * *$ & $279.21 * *$ \\
\hline PGYC-3×Pant Khira-1 & $-15.12 * *$ & $6.82 * *$ & $-13.88 * *$ & $18.52 * *$ & $-25.13 * *$ & $29.43 * *$ & $210.00 * *$ & 5.16 & $287.68 * *$ & $287.46 * *$ \\
\hline
\end{tabular}

NNFFF $=$ Node No. of first female flower, DFFFA = Days to first female flower anthesis, DFFH $=$ Days to first fruit harvest, VL $=$ Vine Length, FL = Fruit length, FD = Fruit diameter, NF/P = No. of fruits per plant, AFW = Average fruit weight, FY/P = Yield per plant, FY/ $\mathrm{Ha}=$ Yield per hectare, PCUCP-2=Pant parthenocarpic Cucumber-2, PCUCP-3=Pant parthenocarpic Cucumber-3

\section{References}

1. Airina CK, Pradeepkumar T, George TE, Sadhankumar PG, Krishnan S. Heterosis breeding exploiting gynoecy in cucumber (Cucumis sativus L.). J. Trop. Agric. 2013; 51:144-148.

2. Anonymous. Package of practices for vegetable crops. Directorate of Extension Education, Dr YS Parmar University of Horticulture and Forestry, Nauni, Solan, 2016, 202.

3. Bairagi SK, Singh DK, Ram HH. Studies on heterosis for yield attribute in cucumber (Cucumis sativus L.). Veg. Sci. 2002; 29:75-77.

4. Dogra BS, Kanwar MS. Exploitation of combining ability in cucumber (Cucumis sativus L.). Res. J. Agric. Sci. 2011; 2(1):55-59.

5. Golabadi M, Golkar P, Eghtedary AR. Assessment of genetic variation in cucumber (Cucumis sativus L.) genotypes. Eur. J. Exp. Biol. 2012; 2:826-831.

6. Grafius JE. Heterosis in barley. Agron. J. 1959; 51:551554.

7. Hanchinamani $\mathrm{CN}$, Patil MG. Heterosis in cucumber (Cucumis sativus L.). Asian J. Hort. 2009; 4:21-24.

8. Hayes HK, Jones DF. First generation crosses in cucumber. Conn. Storrs Agril. Stat. 1916; 40:319-322.

9. Hayes HK, Immer FR, Smith DC. Methods of Plant Breeding, McGraw Hill Book Co. Inc., New York, 1955, $52-65$.

10. Hossain MF, Rabbani MG, Hakim MA, Amanullah AS. M, Ahsanullah ASM. Studies on variability, character association and yield performance of cucumber (Cucumis sativus L.). Bangladesh Res. Publications J. 2010; 43:297-311.

11. Hutchins AE. Some example of heterosis in cucumber (Cucumis sativus L.). Proc. Am. Soc. Hort. Sci. 1939; 36:660-664.

12. Jat GS, Munshi AD, Behera TK, Choudhary H, Dev B. Exploitation of heterosis in cucumber for earliness, yield and yield components utilizing gynoecious lines. Indian J. Hort. 2015; 72:494-499.

13. Kumar A, Kumar S, Pal KA. Genetic variability and character association for yield and yield traits in cucumber. Indian J. Hort. 2008; 65:423-428.

14. Kumar J, Munshi AD, Kumar R, Sureja AK. Studies on heterosis in slicing cucumber. Indian J. Hort. 2010; 67:97-201.

15. Kumar J, Munshi AD, Kumar R, Sureja AK, Sharma RK. Combining ability and its relationship with gene action in slicing cucumber. Indian J. Hort. 2011; 68(4): 507-511.

16. Kumar S, Kumar D, Kumar R, Thakur KS, Dogra BS. Estimation of genetic variability and divergence for fruit yield and quality traits in cucumber (Cucumis sativus L.) in North-Western Himalayas. Univers. J. Plant Sci. 2013; 1(2):27-36.

17. Kumar S, Kumar R, Kumar D, Gautam N, Dogra RK, Mehta DK et al. Parthenocarpic gynoecious parental lines of cucumber introduced from Netherlands for developing high-yielding, quality hybrids. J. Crop Improvement. 2016; 30:352-369.

18. Kumar S, Kumar R, Kumar D, Gautam N, Singh N, Parkash $\mathrm{C}$ et al. Heterotic potential, potence ratio, combining ability and genetic control of yield and its contributing traits in cucumber (Cucumis sativus L.). New Zealand J. Crop Hort. Sci, 2017. DOI:10.1080/01140671.2016.1270336

19. Kushwaha ML, Yadav LB, Maurya RP. Heterobeltiosis and combining ability in cucumber (Cucumis sativus L.) under mid hilly area of Uttarakhand. Prog. Agric. 2011; 11:103-107.

20. Musmade AM, Kale PN. Heterosis and combining ability in cucumber (Cucumis sativus L.). Veg. Sci. 1986; 13:60-68

21. Mohanty BK, Mishra RS. Studies on heterosis for yield and yield attributes in Pumpkin (Cucurbita moschata duc. Ex Poir.). Indian J. Hort. 1999; 56:171-178.

22. Moll RH, Stuber CW. Quantitative genetics empirical results relevant to plant breeding. Adv. Agron. 1974; 26:277-313.

23. Munshi AD, Panda B, Behera TK, Kumar R, Bisht IS, Behera TK et al. Genetic variability in Cucumis sativus var. hardwickii R. germplasm. Cucurbit Genetics Cooperative. 2007; 30:5-10.

24. Pandey P, Singh B, Singh M, Rai, M. Heterosis in cucumber (Cucumis sativus L.). Veg. Sci. 2005; 32:143-145.

25. Reddy KAN, Munshi AD, Behera TK, Sureja AK, Sharma RK. Studies on combining ability in cucumber. Indian J Hort. 2014; 71:349-353.

26. Sherpa P, Seth T, Shende VD, Pandiarana N, Mukherjee S, Chattopadhyay A et al. Heterosis, dominance estimate and genetic control of yield and post-harvest quality traits of tomato. J. Appl. Nat. Sci. 2014; 6:625-632.

27. Singh A, Ram HH. Stability analysis for flowering and maturity traits in cucumber (Cucumis sativus L.). Veg. Sci. 2010; 37:210-212.

28. Singh SK, Kishor GR, Srivastava JP. Commercial exploitation of hybrid vigour in cucumber (Cucumis sativus L.). Int. J. Plant Sci. 2012; 7:313-315.

29. Singh JP, Gill HS. Studies on hybrid vigour in cucumber. Indian J. Hort. 1970; 27:36-38.

30. Thakur M, Kumar R, Kumar S. Estimation of heterosis for earliness and yield contributing traits in cucumber (Cucumis sativus L.). Bioscan. 2017; 12(2):1189-1194. 
31. Turner JM. A Study of heterosis in upland cotton II. Combining ability and inbreeding effects. Agron J. 1953; 43:487-490.

32. Yadav YC, Kumar S, Bisen B, Dixit SK. Genetic variability, heritability and genetic advance for some traits in cucumber. Indian J. Hort. 2009; 66:488-491.

33. Vijayakumari P, More TA, Sheshadri VS. Evaluation of gynoecious F1 hybrids for horticultural characters in cucumber. Veg. Sci. 1991; 18:167-176. 